\title{
TIME LAGS IN COMPACT OBJECTS: CONSTRAINTS ON THE EMISSION MODELS
}

\author{
Juri Poutanen \\ Stockholm Observatory, SE-133 36, Saltsjöbaden, Sweden
}

\begin{abstract}
Accreting black holes and neutron stars in their hard (low) state show not only very similar $\mathrm{X} / \gamma$-ray spectra but also that the behaviour of their light curves is quite similar which can be quantified as having similar power-density spectra and Fourier-frequency-dependent time/phase lags. Taken together this argues for a common mechanism of the $\mathrm{X} / \gamma$-ray production in these objects. This mechanism is probably a property of the accretion flow only since it does not depend on the nature of the compact object. In this paper, I review the observational data paying most attention to the properties of the temporal variability such as the time/phase lags that hopefully can help us to discriminate between different theoretical models. I also discuss the models developed to account for the basic observational facts. Particularly, I show that the commonly used Compton cloud models with constant temperature cannot explain variable sources without violating the energy conservation law. Alternative models where time lags are related to the spectral evolution during X-ray flares are discussed and compared with observations. Compton reflection from the outer edge of the accretion disc is shown to markedly affect the time lag Fourier spectrum.
\end{abstract}

KEYWORDS: accretion, accretion discs; black hole physics; stars: neutron; stars: flare; stars: individual (Cygnus X-1); X-ray: stars.

\section{MODELS FOR THE FORMATION OF X/ $\gamma$-RAY SPECTRA}

X-ray and gamma-ray spectra of accreting black holes and neutron stars are deconvolved into (at least) two components: a soft component interpreted as emission from an optically thick accretion disc, and a hard tail associated with a hot (10-100 $\mathrm{keV}$ ) "corona". Reviews of the spectral properties of Galactic black hole candidates (GBHs) can be found in Gilfanov et al. (1995), Tanaka \& Lewin (1995), Grebenev et al. (1993, 1997), Grove et al. (1997), and Poutanen (1998). X/ $\gamma$-ray properties of radio-quiet active galactic nuclei (AGN) are reviewed by Zdziarski et al. (1997), Johnson et al. (1997), and Zdziarski (1999). Recent results on the broad-band spectra of accreting neutron stars are presented by Barret et al. (2000).

An amusing fact is that super-massive black holes in AGN, GBHs and accreting neutron stars in their hard (low) states (see Tanaka \& Lewin 1995; Gilfanov et al. 1995 for the definition of the spectral states) show very similar $\mathrm{X} / \gamma$-ray spectra (see Zdziarski 1999; Barret et al. 2000). Furthermore, properties of their rapid temporal 
variability are also similar (van der Klis 1995b; Wijnands \& van der Klis 1999; Psaltis, Belloni \& van der Klis 1999; Ford et al. 1999; Edelson \& Nandra 1999; Chiang et al. 2000). All this argues for a common mechanism of the X-ray production in all these sources.

There are good reasons to believe that the main radiative mechanism for the production of the hard X-rays is Comptonization of soft photons (e.g., Shapiro, Lightman, \& Eardley 1976; Sunyaev \& Trümper 1979; Sunyaev \& Titarchuk 1980). However, it is not completely clear what determines the observed spectral slopes. The geometry of the X-ray emitting region and the source of soft photons is still a matter of debate (see Svensson 1996; Poutanen 1998; Beloborodov 1999b; Wardzinski \& Zdziarski 2000).

An important clue to our understanding of the X-ray production came from the discovery of Fe lines (at $\sim 6.4 \mathrm{keV}$ ) and the hardening of the spectra above $10 \mathrm{keV}$ in AGN (Pounds et al. 1990; Mushotzky, Done, \& Pounds 1993; Nandra \& Pounds 1994), Cygnus X-1 (e.g., Done et al. 1992; Gierliński et al. 1997), and neutron stars (e.g., Yoshida et al. 1993). These features are associated with the reflection of hard X-rays from cold material (Basko, Sunyaev, \& Titarchuk 1974; George \& Fabian 1991; Magdziarz \& Zdziarski 1995; Poutanen, Nagendra, \& Svensson 1996). These observations gave support to the so called two-phase accretion disc-corona models. In such models, X-rays are emitted by a hot rarified corona above the cold accretion disc (Haardt \& Maraschi 1993; Haardt, Maraschi, \& Ghisellini 1994; Stern et al. 1995; Poutanen \& Svensson 1996). Hard X-rays from the corona, being reprocessed in the cold disc, produce the reflection hump as well as most of the seed soft photons that are subsequently Comptonized to produce the hard X-rays. This is the feedback mechanism. The geometry of the corona determines the feedback factor which in its turn determines the spectral slope of the escaping radiation. The temperature of the emitting plasma (or to be more exact, the Kompaneets $y$-parameter) is determined by the energy balance between heating (by magnetic reconnection?) and cooling (by Comptonization of soft photons).

Further support for the feedback models was recently given by Zdziarski, Lubinski, \& Smith (1999) (see also Zdziarski 1999; Gilfanov, Churazov, \& Revnivtsev $2000)$ who found a correlation between the amount of reflection $(R \equiv \Omega /(2 \pi)$, where $\Omega$ is a solid angle subtended by cold material as viewed from the X-ray source) and the intrinsic photon spectral index, $\Gamma$, of the hard X-ray component. Such a correlation can easily be explained if there is overlap between the hot corona and the cold disc (Poutanen, Krolik, \& Ryde 1997). The further the cold disc penetrates into the corona, the larger is the cooling, the smaller is the temperature of the corona, the softer is the spectrum, and, finally, the larger is the amplitude of the reflection. The model, however, appears to have trouble giving reflection amplitudes above $R_{\max } \sim 0.5$ (if the coronal optical depth $\tau_{\mathrm{T}} \sim 1$, see Zdziarski et al. 1997) due to partial smearing of the reflection component by the hot corona.

Alternatively, the observed $R-\Gamma$ correlation can be reproduced by variations of the bulk velocity of the $\mathrm{X} / \gamma$-ray emitting plasma (Beloborodov 1999a,b). If the emitting regions are sufficiently compact to produce electron-positron pairs, the 
pressure of the radiation reflected and reprocessed in the disc accelerates pairs to mildly relativistic velocities away from the disc. On the other hand, for proton dominated plasmas, a small anisotropy in the energy dissipation mechanism can result in the ejection of particles away or towards the disc. Ejection away from the disc reduces $R$ below 1 and leads to hard spectra, while ejection towards to the disc can result in apparent $R>1$ as is observed in some objects.

The physical possibility of the corona formation was studied by Galeev, Rosner, \& Vaiana (1979). They showed that the magnetic fields, being amplified in the cold disc due to turbulent motions and differential rotation, do not have time to annihilate inside the disc on the inflow time scale. Instead, the field loops are expelled from the disc by buoyancy (the Parker instability) and they annihilate in the tenuous corona. Beloborodov (1999a) showed that the mechanism studied by Galeev et al. is able to produce a corona of limited luminosity which is $h / r$ (the ratio of the disc height to its radius) times smaller than the disc luminosity. By contrast, in some sources most of the energy escapes in the form of hard X-rays. Beloborodov also argued that the magneto-rotational instability (Velikhov 1959; Chandrasekhar 1960; Balbus \& Hawley 1991) increases the rate of the magnetic field generation (as compared with the Galeev et al. model) thus producing an active magnetic corona where a large fraction of the gravitational energy can finally be dissipated in magnetic flares. These qualitative arguments were recently supported by numerical three-dimensional magnetohydrodynamical simulations of Miller \& Stone (2000) who showed that about $25 \%$ of the total energy dissipation can occur in the rarified corona.

An alternative to the magnetic corona is the hot disc model (Shapiro et al. 1976; Ichimaru 1977; Narayan, Mahadevan, \& Quataert 1998; Zdziarski 1998; Esin et al. 1998) which is also able to explain the observed $\mathrm{X} / \gamma$-ray spectra. In order to distinguish between the models, it would be helpful to compare the predictions of different models with the temporal variability data (see van der Klis 1995a,b and Cui 1999a for recent reviews). Unfortunately, most of the papers on the spectral models do not consider the temporal variability. On the other hand, most of the models designed to explain the temporal variability data do not pay enough attention to the emission processes and the physics of the spectral formation.

In this review, we will discuss the variability data keeping in mind recent advances in modelling broad-band $\mathrm{X} / \gamma$-ray spectra of accreting black holes and neutron stars. Most attention will be paid to the time lags that can shed light on the mechanism of the X-ray production. Then we discuss simple phenomenological models that are able to explain some of the observational facts. After that, we switch to the physical models. In particular, the properties of the Comptonizing regions will be discussed. We will point out the flaws in models that do not consider the energy balance in the "Compton cloud", and then discuss models that satisfy the energy conservation law and confront them with the available data. 


\section{OBSERVING TIME LAGS IN ACCRETING BLACK HOLES AND NEU- TRON STARS}

The standard temporal characteristics that are usually computed are the powerdensity spectra (PDS) in different energy channels, auto/cross-correlation functions $(\mathrm{ACF} / \mathrm{CCF})$, the time/phase lags between the variability in different energy channels, the coherence function, etc. Time lags have been studied by two methods, by constructing the CCF and by cross-spectral analysis (for details, see Lewin et al. 1988; van der Klis 1989; Nowak et al. 1999a).

\subsection{Lags in Black Hole Sources}

The observations of Cygnus X-1 from sounding rockets and HEAO 1 (Priedhorsky et al. 1979; Nolan et al. 1981) showed that the CCF between different energy channels peaks very close to zero lag (delay $\lesssim 40 \mathrm{~ms}$ ), but it is slightly asymmetric. Similar asymmetry was found in the EXOSAT data by Page (1985) who claimed a $6 \pm 1 \mathrm{~ms}$ shift of the peak of the CCF between the $5-14 \mathrm{keV}$ and the $2-5 \mathrm{keV}$ bands. Recent $R X T E$ observations clearly show asymmetries of the CCFs, which, however, peak within $\sim 1 \mathrm{~ms}$ from zero lag (see Fig. 1). This suggests that the relation between the variation in the two bands are not simply a time delay. Asymmetry is also observed in the RXTE data of GX 339-4, where the CCFs are offset by $\lesssim 5$ ms from zero (using the 2-5 and 10-40 keV bands, Smith \& Liang 1999). The CCFs of AGN also display similar properties (e.g., Papadakis \& Lawrence 1995; Lee et al. 1999).

The CCFs cannot be fitted with simple exponentials at any time scale. A reasonably good description of the $\mathrm{CCF}$ is in terms of a stretched exponential $C C F(t)=\beta \exp \left[-\left(\left|t-t_{0}\right| / \tau\right)^{\nu}\right]$ (see the inset of Fig. 1), where the normalisation $\beta \approx 1$, the time where the CCFs peak $t_{0}<10^{-3} \mathrm{~s}, \nu \sim 2 / 3$ in the range $|t|<0.3 \mathrm{~s}$, and the time constant $\tau$ is different for rising and decaying part of the CCF. Such a behaviour is probably the result of self-similarity of the light curve. It is interesting to note that the ACF of gamma-ray bursts also show a similar stretched exponential behaviour (see Stern \& Svensson 1996; Beloborodov 1999c).

Since the CCF does not show which frequencies contribute most to the observed lags, van der Klis et al. (1987) suggested to use instead the cross-spectrum for such an analysis. In the hard state of Cyg X-1 observed by the Ginga satellite, the time lags between the variability in the 1.2-4.7 and 4.7-9.3 keV energy bands reached $0.1 \mathrm{~s}$ and had a strong Fourier-frequency dependence $\delta t(f) \sim f^{-1}$, i.e., the phase lag $\delta \phi(f) \approx$ const (Miyamoto et al. 1988; Miyamoto \& Kitamoto 1989). Similar lags were observed in other GBHs, GX 339-4 and GS 2023+338 (三V404 $\mathrm{Cyg}$ ), in their hard state (Miyamoto et al. 1992). The analysis of the RXTE data for Cyg X-1 (Nowak et al. 1999a), GX 339-4 (Nowak et al. 1999b), 1E 1740.7-2942 and GRS 1758-258 (Smith et al. 1997), and GS 1354-644 (Revnivtsev et al. 2000)

\footnotetext{
${ }^{1}$ The cross-spectrum $C(f) \equiv S^{*}(f) H(f)$, where $S(f)$ and $H(f)$ are the Fourier transforms of the light curves in the soft and hard energy channels, respectively. The phase lag, $\delta \phi(f) \equiv \arg [C(f)]$, and the time lag, $\delta t(f) \equiv \delta \phi(f) /(2 \pi f)$. The lags are positive when hard photons are lagging the soft ones.
} 


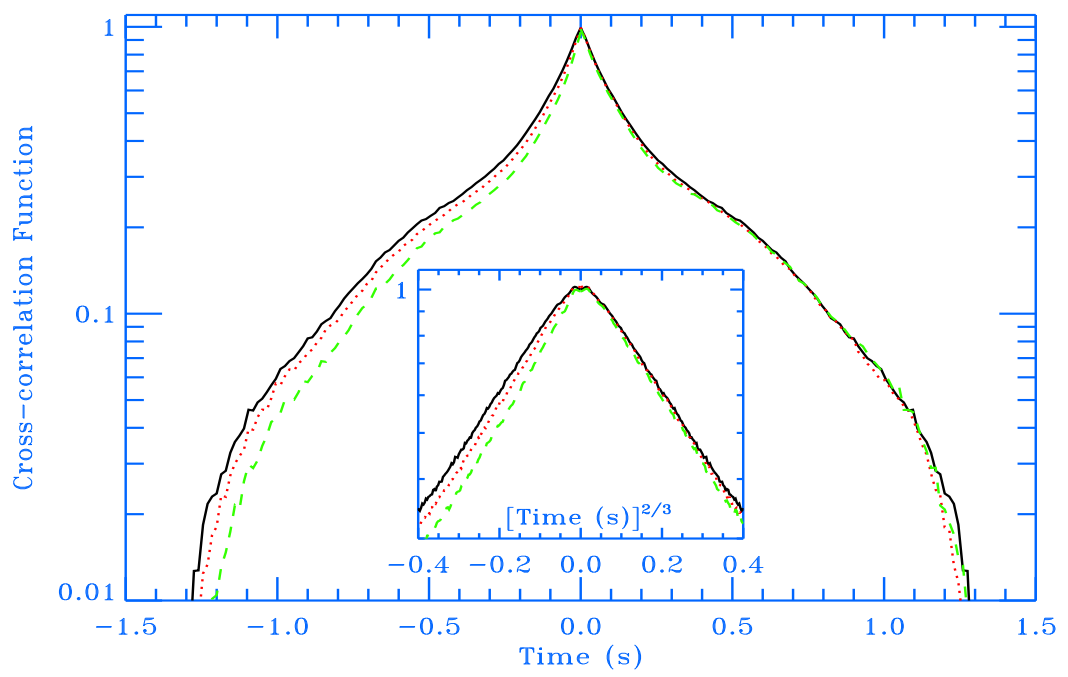

FIGURE 1. The cross-correlation functions of Cyg X-1 in the hard state (RXTE observations from October 22,1996$)$. The solid curves are the autocorrelation function for the 2-3.9 keV energy channel, the dotted and dashed curves are the CCFs for the 6-8.2 and 14-70 keV vs. the 2-3.9 keV energy channel, respectively. Note, that CCFs in the hard state sometimes have much broader wings extending to $\sim 8 \mathrm{~s}$ (e.g., Nolan et al. 1981; Maccarone et al. 2000).

confirming the general features seen in the Ginga data, showed more complicated behaviour of the phase lag spectra which have a number of shelves and breaks (see Fig. 2).

Grove et al. (1998) extended this analysis to higher energies with the data from CGRO/OSSE. The time lags at low frequencies reached $0.3 \mathrm{~s}$ between the 50-70 and the $70-100 \mathrm{keV}$ photons in the light curve of GRO J0422+32 (三Nova Persei 1992). The breaks detected in the time lag spectrum at $0.1 \mathrm{~Hz}$ may be related to the quasi-periodic oscillation (QPO) observed at $0.23 \mathrm{~Hz}$. The CGRO/BATSE data of Cyg X-1 (Crary et al. 1998), GRO J0422+32 and GRO J1719-24 (三Nova Oph 1993) (van der Hooft et al. 1999a,b) show very similar time lag spectra.

The time lags of GBHs in their soft state (when $\Gamma \approx 2.5$ ) turn out to be somewhat different. Soft lags were observed between the $1.2-2.3 \mathrm{keV}$ and the $2.3-4.6 \mathrm{keV}$ bands in GX 339-4 (Miyamoto et al. 1991) and GS 1124-68 (三Nova Muscae 1991; see Miyamoto et al. 1993; Takizawa et al. 1997), while the higher energy photons were lagging the variability in the 2.3-4.6 keV band. In one observation of GS 1124-68, the variability in the 4.6-9.2 $\mathrm{keV}$ was the most advanced. The phase lag reached $\sim 1 \mathrm{rad}$ which is much larger than the lags in the broad band noise observed in the GBHs in their hard state. Rapid time variations were mostly due to the harder power-law component which is clearly seen from the rms amplitude. It is interesting that the 


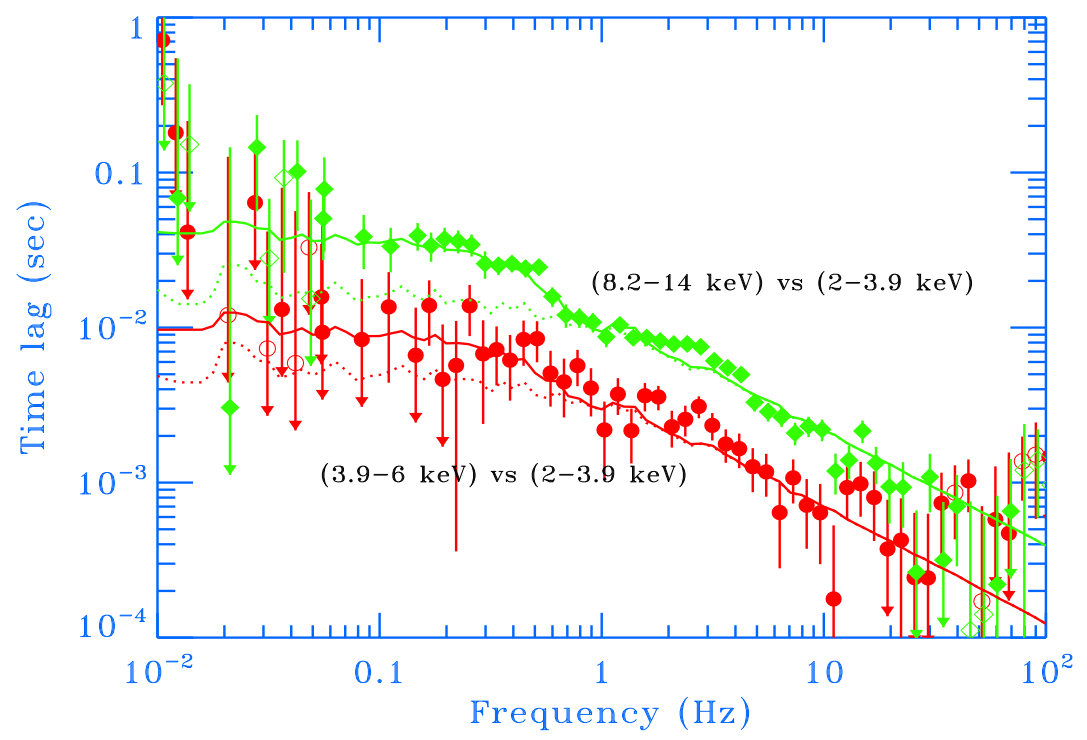

FIGURE 2. Time lags between signals in the $8.2-14 \mathrm{keV}$ and the $3.9-6 \mathrm{keV}$ bands vs the 2-3.9 keV band in Cyg X-1 (RXTE observations from October 22, 1996). The dotted curves are the model of Poutanen \& Fabian (1999b) with the maximum flare time scale of $\tau_{\max }=0.2 \mathrm{~s}$. The solid curves show the same model with $70 \%$ of the observed Compton reflection produced at a distance of $t_{\text {ref }} \sim 1$ light seconds from the central X-ray source. The reflector acts as a low pass filter so that additional delays appear only at frequencies $f \lesssim 1 / t_{\text {refl }}$, and only at the energies where reflection is significant. See $\S 4.2$ for details.

lags seem to saturate above $10 \mathrm{keV}$. It is worth pointing out that the largest lags here are observed at the QPO frequency. On the other hand, the time lag spectrum of Cyg X-1 in the soft state looks quite similar to that in the hard state (Cui et al. 1997).

Time lags have been observed in GRS $1915+105$ in the $67 \mathrm{mHz}$ QPO by Cui (1999b) and in the broad-band noise and QPOs by Reig et al. (2000). The lags show a very complicated structure, sometimes changing signs from one harmonic to another, and the sign also depends on the frequency of the QPO. Wijnands, Homan, \& van der Klis (1999) and Cui, Zhang, \& Chen (2000) observed lags in the broad-band noise and the QPOs of XTE J1550-564.

\subsection{Lags in Neutron Star Sources}

Hard time lags in neutron star sources were discovered by Hasinger (1987) in the CCF of Cyg X-2 (comparing the 1-5 and the $5-17 \mathrm{keV}$ bands) in its horizontal branch (HB, see Lewin et al. 1988 for definitions of branches). The CCF also had sinusoidal oscillations due to the QPO. The time lags showed anti-correlation with the QPO 
frequency, dropping from $4 \mathrm{~ms}$ to $1.5 \mathrm{~ms}$ when the QPO changed from $20 \mathrm{~Hz}$ to 50 $\mathrm{Hz}$. Hasinger interpreted the lags as delays due to scattering (Comptonization) in the hot cloud and the anti-correlation as an indication of a change in the system size. Associating the QPO frequency with the Keplerian frequency at some radius gives the relation, $\delta t(f) \propto f_{Q P O}^{-2 / 3}$, while the actual data are much better described by $\delta t \propto f_{Q P O}^{-1}$, i.e. $\delta \phi=2 \pi f \cdot \delta t=$ const.

Using the cross-spectrum techniques van der Klis et al. (1987) confirmed the existence of $\sim 3 \mathrm{~ms}$ hard lags in the 20-40 Hz QPO of Cyg X-2 (and GX 5-1) and discovered $8 \mathrm{~ms}$ soft lags in the low-frequency noise, which were interpreted as a softening of the spectrum during the shots that cause QPOs. These results were confirmed by Vaughan et al. (1994) who also showed (from the analysis of the Ginga data of GX 5-1 on the HB) that the time lags increase with photon energy.

In both Cyg X-2 and GX 5-1 in their normal branch, the lags at the $\sim 5 \mathrm{~Hz}$ QPO showed energy dependence (Mitsuda \& Dotani 1989; Vaughan et al. 1999) reaching $\delta \phi \sim \pi \operatorname{rad}$ (i.e., $\delta t \sim 0.2 \mathrm{~s}$ ) for $10 \mathrm{keV}$ photons vs $2 \mathrm{keV}$. At the same time, the rms amplitude of the QPO in Cyg X-2 had a minimum at $5 \mathrm{keV}$ and in GX 5-1 it increased above $2.5 \mathrm{keV}$. This behaviour can be interpreted as a pivoting of the spectrum around $3-5 \mathrm{keV}$.

With the larger effective area of RXTE, Ford et al. (1999) and Olive \& Barret (2000) discovered phase lags in the broad-band noise of three atoll sources, 4U0614+09, 4U1705-44, and 4U1728-34. These lags are very similar to those in GBHs like Cyg X-1 and GX 339-4, which tells us that the mechanism responsible for the lags does not depend on the presence or absence of the hard surface of the neutron star, magnetosphere, boundary layer, etc., but instead is a property of the accretion flow.

A number of neutron stars show $\mathrm{kHz}$ QPOs in their light curves as revealed by $R X T E$. Kaaret et al. (1999) find $25 \mu$ s soft lags between the $4-6 \mathrm{keV}$ and the $>9 \mathrm{keV}$ photons in the $800 \mathrm{~Hz}$ QPO in the atoll source 4U1636-536. Analysing the $550 \mathrm{~Hz}$ oscillations of Aquila X-1, Ford et al. (1999) found soft lags, $\delta \phi \sim 1$ $\mathrm{rad}$, between the $3-6 \mathrm{keV}$ and the $>6 \mathrm{keV}$ photons. Similar lags were found in the accreting millisecond pulsar SAX J1808.4-3658 (Cui, Morgan, \& Titarchuk 1998; Ford 2000). The lags in the $830 \mathrm{~Hz}$ QPO in $4 \mathrm{U}$ 1608-52 (Vaughan et al. 1997, 1998) reach $60 \mu$ s between the 5 and the $25 \mathrm{keV}$ photons.

\section{PHENOMENOLOGICAL ENERGY DEPENDENT SHOT NOISE MODELS}

It is clear that the observed zoo of time lags cannot be explained by any single model. Different mechanisms should be involved in producing the lags at different Fourier frequencies, the lags in the QPOs and the coherent pulsations, and the lags in the broad-band noise.

Let us first consider the simplest possible model that produces time lags: a shot noise model (Terrell 1972), where shots (=flares) are uncorrelated with each other. We assume that the shot time profiles at different energies have the same shape, but slightly different time constants. As an example we take a shot profile at soft 
energies $s(t)=[t / \tau]^{p} \exp [-t / \tau]$ and at hard energies $h(t)=[t /(\eta \tau)]^{p} \exp [-t /(\eta \tau)]$, where $t>0$ is measured from the beginning of the shot and $p$ is positive. The Fourier transforms, $S(f)$ and $H(f)$, are $S(f) \propto \tau \Gamma(p+1) /(1-i 2 \pi f \tau)^{p+1}$ and $H(f) \propto \eta \tau \Gamma(p+1) /(1-i 2 \pi f \eta \tau)^{p+1}$. The PDSs are $\propto|S(f)|^{2}$ and $|H(f)|^{2}$. For small frequencies, $f \ll 1 /(2 \pi \tau)$, the PDSs have a flat dependence on frequency, $\propto f^{0}$, while for large frequencies, $f \gg 1 /(2 \pi \tau)$, the PDSs decay as $f^{-2(p+1)}$. The power per logarithm of frequency (i.e., $f \times P D S(f)$ ) peaks for soft photons at $f_{s, \max }=$ $1 /[2 \pi \tau \sqrt{2 p+1}]$ and at $f_{h, \max }=1 /[\eta 2 \pi \tau \sqrt{2 p+1}]$ for hard photons. The phase lags $\delta \phi(f)=(p+1)[\arctan (\eta 2 \pi f \tau)-\arctan (2 \pi f \tau)]$. The lag is positive when hard photons are lagging soft ones (i.e., for $\eta>1)$. For small frequencies, $\delta \phi(f)$ rises as $\approx(p+1)(\eta-1) 2 \pi f \tau$, while for large frequencies, it decays as $(p+1)(\eta-1) /(\eta 2 \pi f \tau)$. The lag reaches a maximum of $\delta \phi_{\max }=2(p+1)(\arctan \sqrt{\eta}-\pi / 4)$ at $f=1 /(2 \pi \tau \sqrt{\eta})$ close to $f_{s, \max }$ and $f_{h, \max }$, the frequencies where $f \times P D S_{s, h}(f)$ peak.

One can also consider a modified shot noise model, where the shot time scales are distributed according to a power law, $\rho(\tau) \propto \tau^{-p}$ between $\tau_{\min }$ and $\tau_{\max }$ (see, e.g., Miyamoto \& Kitamoto 1989; Lochner, Swank, \& Szymkowiak 1991), with the same ratio $\eta$. Physically this could correspond to, for example, the situation when flares of different durations appear at different radii from the central object (Poutanen \& Fabian 1999a). A power-law distribution of $\tau$ assures that the PDS is also a powerlaw $\propto f^{-(3-p)}$ (Lochner et al. 1991). If the flares are self-similar, then the phase lag will be constant $\approx \delta \phi_{\max }$ for $f \gg f_{\min } \equiv 1 /\left(2 \pi \tau_{\max }\right)$ and $f \ll f_{\max } \equiv 1 /\left(2 \pi \tau_{\min }\right)$, decay as $1 / f$ at $f>f_{\max }$ and rise linearly at $f<f_{\min }$. The corresponding time lags are constant, $\delta t_{\max }=2 \pi \tau_{\max } \delta \phi_{\max }$, for $f<f_{\min }$, and decay approximately as $1 / f$ between $f_{\min }$ and $f_{\max }$. Note that in this model the coherence function (Vaughan \& Nowak 1997; Nowak et al. 1999a) is close to unity, since the light curves at different energies are almost perfectly synchronised.

If we assume that $\eta>1$, there are hard lags and the predicted behaviour of the time lags and coherence function is in a very good agreement with the observations of GBHs (Poutanen \& Fabian 1999a). However, this model contradicts the CCF of Cyg X-1 (see Fig. 11 and Maccarone et al. 2000). The CCF becomes narrower at larger energies which requires the shots to be narrower at larger energies. If one, however, reverses the time profiles of the shots, so that they rise slower and decay faster (e.g., $\left.s(t)=(-t / \tau)^{p} \exp (t / \tau), t<0\right)$, and one assumes $\eta<1$ (i.e., hard shots are narrower), the CCFs and the time lags can be reproduced, simultaneously.

Much more complicated models which also account for lags in the QPOs sources were developed by Shibazaki et al. (1988). We just note here that if a signal consists of shots appearing almost periodically and if shots at different energies are shifted in time one against another (or, e.g., the minima are reached at the same time and the peaks are not), the phase lag has a very complicated dependency on frequency (e.g., changes sign from one harmonic to another) depending on the shot profiles. 


\section{PHYSICAL MECHANISMS FOR PRODUCING LAGS}

\subsection{Static Compton Cloud Models}

Since Comptonization is the most probable mechanism for X-ray production in compact objects, it is natural to attribute the time delays between hard and soft photons to this process. Hard photons are the result of more scattering and so emerge after, or lag behind, softer ones. Consider a static "Compton cloud" with fixed Thomson optical depth, $\tau_{\mathrm{T}}$, and electron temperature $\Theta=k T_{e} / m_{e} c^{2}$. A soft seed photon of energy $E_{0}$ injected into the cloud increases its energy by a factor of $A_{1}=1+4 \Theta+16 \Theta^{2}$ on average after each scattering, so that after $N$-scatterings its energy $E_{N}=A_{1}^{N} E_{0}$. The photon mean free path is $\lambda \approx R / \max \left(1, \tau_{\mathrm{T}}\right)$ (where $R$ is the size of the $\mathrm{X}$-ray producing region, and where we accounted for the fact that we are interested only in those photons that actually have undergone scatterings in the cloud). The time between successive scatterings is then $t_{c}=R /\left(c \max \left[1, \tau_{\mathrm{T}}\right]\right)$, so the time needed to reach the energy $E_{N}$ is (Sunyaev \& Titarchuk 1980; Payne 1980)

$$
t_{N}=N t_{c}=\frac{R / c}{\max \left(1, \tau_{\mathrm{T}}\right)} \frac{\ln \left(E_{N} / E_{0}\right)}{\ln A_{1}},
$$

which translates to $t_{N} \sim 10^{-4} \mathrm{~s}$ for $k T_{e} \sim 50 \mathrm{keV}, \tau_{\mathrm{T}} \sim 1, R=10 \mathrm{~km}$, and $E_{N} / E_{0} \sim 10$.

This model was criticised by Miyamoto et al. (1988), Miyamoto et al. (1991) and Vaughan et al. (1994). First, the large size of the cloud $\left(10^{3}-10^{5} R_{g}\right.$, where $R_{g}$ is the Schwarzschild radius, $2 G M / c^{2}$ ) is needed to produce large delays observed in GBHs and neutron stars. Such cloud is physically unrealistic, since most of the gravitational energy is dissipated within $10 R_{g}$. Second, the lags predicted by the model are independent of the Fourier frequency (Miyamoto et al. 1988) contrary to the observed $\sim 1 / f$ dependence. Third, it is assumed that the soft photons produce the variability, while the hot cloud is not variable. Observationally, it is well established that when a soft black body spectrum is observed in GBHs it is much less variable than the hard X-rays (e.g. Miyamoto et al. 1991), so that the hard X-ray variability is most probably intrinsic to the hot cloud itself.

Finally, we would like to point out that due to the requirement of energy conservation the whole concept of a static Compton cloud with a constant temperature is physically unrealistic. The total emitted X-ray luminosity (produced by Comptonization of soft radiation) is $L_{\text {tot }}=L_{h}+L_{s}$, where $L_{h}$ is the heating rate in the hot cloud and $L_{s}$ is the luminosity of seed soft photons. For hard spectra (i.e. $\left.L_{h} \gg L_{s}\right), L_{\text {tot }} \approx L_{h}$. The total X-ray luminosity is thus a function of the heating rate only and it does not depend on the amount of seed soft photons. By changing $L_{s}$, one effectively changes the spectral slope of the emergent X-ray radiation which is a function of the Compton amplification factor $A \equiv L_{h} / L_{s}$. This results in the pivoting of the spectrum (see Poutanen 1998; Beloborodov 1999b) without a noticeable increase in $L_{\text {tot }}$. The larger the $L_{s}$, the smaller the equilibrium temperature of the emitting electrons, and the softer the spectrum. By contrast, in static 
Compton cloud models no changes in the electron temperature are considered in reaction to the changes in the number of soft photons, violating thus the energy conservation law. In order to increase the emitted luminosity, one has to change the energy dissipation rate in the cloud, but then exactly these changes will be driving the variability.

Recently, Kazanas, Hua, \& Titarchuk (1997) (see also Böttcher \& Liang 1998; Hua, Kazanas, \& Cui 1999) modified the Comptonization model. Instead of a homogeneous Compton cloud, the density profile, $n(r) \sim 1 / r$, was assumed (in this case, one has equal optical depth per logarithm of radius). The variability is still driven by changing the rate of soft photon injection in the center of the cloud. Then larger radii produce lower frequency variability (filtering out high frequency signal) and larger lags, while the smaller radii produce higher frequency variability and smaller lags (see also Nowak et al. 1999a,c). This model solves only one of the problem mentioned above $(1 / f$ time lag dependence), while the other problems remain unsolved. Another modification of the model was considered by Böttcher \& Liang (1999) based on an earlier suggestion by Miyamoto et al. (1988). Here, small cold clouds are assumed to free-fall into the hot central cloud thus changing the input of soft photons. Again, this model has problems with energy conservation.

\subsection{The Dynamic Compton Cloud}

Miyamoto et al. (1988) pointed out that some modulation mechanism must be invoked to produce the strongly frequency-dependent time lags. Poutanen \& Fabian $(1999 \mathrm{a}, \mathrm{b})$ proposed a model where the time lags are produced by the evolution of the flare spectrum. They assumed that the energy dissipation varies in time. For a small (of the order of $R_{g}$ ) emitting region (ER) one can consider the spectral evolution as a sequence of steady-states as long as the characteristic time scale of variability is $\tau \gg R / c$. Any changes in the amplification factor $A$ would cause spectral variability and, specifically, a continuous increase of $A$ with time during the course of the flare would cause a soft-to-hard spectral evolution producing hard time lags of the order of the flare time scale (see $\S$ \&.). Poutanen \& Fabian (1999b) considered three mechanisms that can increase $A$.

(1) A flare starts in the background of soft photons. The X-ray spectrum is soft as long as $L_{h}<L_{s, \mathrm{bkg}}$. With increasing $L_{h}$, the soft photon input gets dominated by reprocessed photons. The spectral slope is then determined by the feedback parameter $D \approx 1 / A$ (the fraction of $L_{h}$ returned to the ER after the reprocessing in the disc into soft seed photons, see Stern et al. 1995; Svensson 1996; Beloborodov 1999b) and the spectrum becomes hard.

(2) The dissipation is accompanied by pumping net momentum into the hot plasma of the emission region (Beloborodov 1999a,b). The resulting bulk velocity increases with increasing luminosity. It leads to a lower feedback and higher $A$ (if the velocity is directed away from the disc).

(3) The differential rotation of the footpoints of a magnetic loop at the disc surface causes a twisting and elevation of the loop (Romanova et al. 1998). The time scale of 
the evolution is of the order of the Keplerian time-scale. When the emission region moves away from the disc, the feedback decreases and $A$ increases (see Fig. 3).

In all these cases the spectral evolution proceeds from soft to hard. The hard time lags between energies $E$ and $E_{0}$ are $\propto \tau \ln \left(E / E_{0}\right)$. If there is a distribution of time scales $\tau$ between, say, $1 \mathrm{~ms}$ and $0.3 \mathrm{~s}$ (e.g., Keplerian time scales at the radii between the innermost radius of the accretion disc and $\left.\sim 50 R_{g}\right)$, the time lags $\propto 1 / f$ at the characteristic frequencies of the variability (see $\S 3$. and Fig. 2).

If $\tau \sim$ a few light crossing time of the ER, the spectrum in the beginning of the flare is hard because of photon starvation (one needs a few $R / c$ to get reprocessed soft photons into the ER) and softens towards the end of the flare (Poutanen \& Fabian 1999a; Malzac \& Jourdain 2000) producing soft time lags. Observing the change of sign of the time lags at some Fourier frequency, $f_{\mathrm{sgn}}$, would determine the size of the ER that produces variability at these frequencies (e.g., for Cyg X-1, $\left.R \lesssim 10 R_{g}\left(30 \mathrm{~Hz} / f_{\mathrm{sgn}}\right)\right)$.
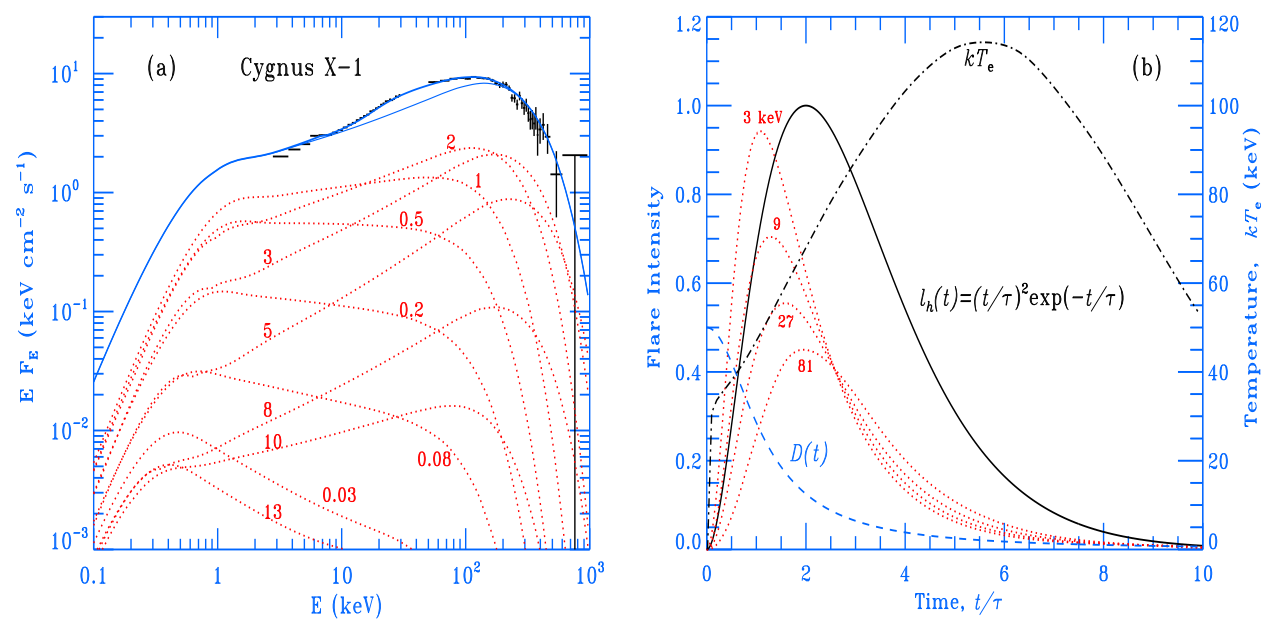

FIGURE 3. (a) Spectral evolution of a magnetic flare. Time resolved spectra (without Compton reflection) are presented by dotted curves; marks are times, $t / \tau$, from the beginning of the flare. The time-averaged Comptonized spectrum is shown by a thin solid curve. The time-averaged spectrum of Cygnus X-1 (simultaneous Ginga and OSSE data from June 1991, the data set \# 1 in Gierliński et al. 1997) is plotted with crosses and the best fit with the flare model with a solid curve $\left(\chi^{2} / \mathrm{dof}=50.0 / 75\right)$. Interstellar absorption is removed when plotting the model spectrum. (b) The flare light curves at 3, 9, 27, and 81 $\mathrm{keV}$ are presented by dotted curves. Solid curve - the heating rate, $l_{h}(t) \propto(t / \tau)^{2} \exp (-t / \tau)$; dashed curve - the feedback factor $D(t)$; dot-dashed curve - the temperature of the emission region. See Poutanen \& Fabian (1999a,b) for details. 


\subsection{Small Scale Spectral Transitions}

The models considered above can explain the time lags in the broad-band noise. In some QPO sources the time lags show a very complicated behaviour (see $\S$ 2.) and may require different explanation. In radiation-hydrodynamic model (see, e.g., Lamb 1989; Miller \& Lamb 1992), QPOs appear as a result of oscillations in the optical depth of the radial flow due to the radiation feedback from the neutron star surface. The resulting spectral pivoting produces phase lags and the increase of the rms amplitude variability above the pivoting point. This model, however, is not applicable to the QPOs and the lags in black holes sources (see, e.g., Takizawa et al. 1997) because of the absence of a hard surface.

The galactic microquasar GRS 1915+105 shows large amplitude oscillations with periods varying from $<1$ up to $100 \mathrm{~s}$. These time scales are a few orders of magnitudes larger than Keplerian time scales and up to $10^{6}$ time larger than the light crossing time of the ER. The best candidate for producing the spectral variability that causes the lags in GRS $1915+105$ is the oscillation of the inner radius of the cold disc on viscous time scales. Such oscillations are similar to the spectral transitions, but have smaller amplitude and occur at shorter time scales. Changes of the relative geometry of the hot corona and the cold disc (with or without changes of the total luminosity) cause spectral pivoting at a few $\mathrm{keV}$ (see, e.g., Poutanen, Krolik, \& Ryde 1997; Esin et al. 1998). The fluxes below and above the pivot point oscillate then with a phase shift of $\sim \pi$. The rms amplitude of the QPO increases with the energy. The phase lags between the energies above the pivot point can then be produced if the oscillations are time asymmetric (see Fig. 11 in Morgan, Remillard, \& Greiner 1997; Vilhu \& Nevalainen 1998).

Similar (but aperiodic) changes in the inner disc radius can be responsible for the broad-band variability observed at $f<1 \mathrm{~Hz}$ in, e.g., Cyg X-1. Associated spectral changes can manifest themselves in time lags observed at these frequencies.

\subsection{Delays due to Compton Reflection and Reprocessing}

The spectra of accreting GBHs and neutron stars show signatures of Compton reflection (see $\S$ 1.). Some fraction of the X-ray photons can be reflected from the outer edge of a flared accretion disc, a wind from the companion, etc. Such a reflector acts as a low pass filter smearing out the high frequency variations and produces lags corresponding to the light travel time to the reflector only at lower frequencies. Such processes can explain the break in the time lag spectra observed in Cyg X-1 (see Fig. 2) and in other GBHs at $f \lesssim 1 \mathrm{~Hz}$.

Reprocessed soft radiation which accompanies Compton reflection is emitted in the optical and UV spectral bands if the reprocessing occurs far away from the central X-ray source. The time delays can then be measured between the optical/UV and the X-ray radiation (e.g., Hynes et al. 1998). On the other hand, reprocessing in the vicinity of the X-ray emitting region, produces time delays of the order of the time scale of the spectral evolution in the hard X-ray band. This may be one reason for the observed soft lags in the soft state of GX 339-4 and GS 1124-68 (§ 2.1.). 


\subsection{Hot Spots on the Neutron Star Surface}

Some neutron star sources show lags in their periodic oscillations at $\mathrm{kHz}$ frequencies. Ford et al. (1999) and Ford (2000) interpreted the soft lags in Aquila X-1 and in the accreting millisecond pulsar SAX 1808.4-3658 using a model of a rotating hot spot with a black body spectrum at the surface of a neutron star where the lags appear due to Doppler effects. The weak energy dependence of the rms amplitude of the oscillations reported by Cui et al. (1998) rules out the black body model for the spectrum used by Ford. Detailed analysis of the pulsations in the time domain by folding techniques by Revnivtsev (1999) revealed that the pulse profile is distorted at different energies, while the minima are reached at the same time (i.e., there are no lags in the normal meaning of this word).

\section{SUMMARY}

Time lags and other temporal variability data provide strong constraints on the models of the X-ray production. It was demonstrated that static Compton cloud models are based on physically unrealistic assumptions. The models invoking spectral evolution of the flare spectrum can fit both the CCF and the time lag Fourier spectra only if (1) the energy dissipation rate increases slowly and decreases rapidly and (2) the flare spectrum evolves from soft to hard. If soft seed photons are produced by reprocessing the hard ones, the change of sign in the time lag spectrum is expected at high frequencies corresponding to the light crossing time of the emission region. The absence of such a change would put constraints on the size of the emitting region.

We also argued that the reflection of hard X-rays from the outer part of the accretion disc produces time delays that we already might have observed in GBHs. If so, the disc should be flared and the break in the time lag Fourier spectra then corresponds to the size of the accretion disc. Of course, such an interpretation is not unique. Alternatively, small scale spectral transitions (e.g., oscillations of the inner radius of the accretion disc at viscous time scales) might produce time lags observed at lower frequencies.

In the case of (quasi-) periodic oscillations from the neutron star sources, we argued that in order to reproduce both the time lags and the energy dependent rms amplitude, the spectrum of the hot spots should not be close to a black-body.

\section{ACKNOWLEDGEMENTS}

This work was supported by the Swedish Natural Science Research Council and the Anna-Greta and Holger Crafoord Fund. I thank Katja Pottschmidt for providing the time lag Fourier spectra and the light curves of Cyg X-1 used in the calculations of the cross-correlation functions. I am grateful to Andrei Beloborodov and Roland Svensson for valuable comments. 


\section{REFERENCES}

Balbus, S.A., Hawley, J.F. 1991, ApJ, 376, 214

Basko, M.M., Sunyaev, R.A., Titarchuk, L.G. 1974, A\&A, 31, 249

Barret, D., Olive, J.F., Boirin, L., Done, C., Skinner, G.K., Grindlay, J.E. 2000, ApJ, in press astro-ph/9911042

Beloborodov, A.M. 1999a, ApJ, 510, L123

Beloborodov, A.M. 1999b, in High Energy Processes in Accreting Black Holes, ASP Conf. Series Vol. 161, ASP, San Francisco, p.295 astro-ph/990110

Beloborodov, A.M. 1999c, in Gamma-Ray Bursts: The First Three Minutes, ASP Conf. Series Vol. 190, ASP, San Francisco, p.47 (astro-ph/9911122)

Böttcher, M., Liang, E.P 1998, ApJ, 506, 281

Böttcher, M., Liang, E.P. 1999, ApJ, 511, L37

Chandrasekhar, S. 1960, Proc. Natl. Acad. Sci. USA, 46, 253

Chiang, J. et al. 2000, ApJ, 528, 292

Crary, D.J. et al. 1998, ApJ, 493, L71

Cui, W., Zhang, S.N., Focke, W., Swank, J.H. 1997, ApJ, 484, 383

Cui, W., Morgan, E.H., Titarchuk, L.G. 1998, ApJ, 504, L27

Cui, W. 1999a, in High Energy Processes in Accreting Black Holes, ASP Conf. Series Vol. 161, ASP, San Francisco, 97 (astro-ph/9809408)

Cui, W. 1999b, ApJ, 524, L59

Cui, W., Zhang, S.N., Chen, W. 2000, ApJ, 531, L45

Done, C., Mulchaey, J.S., Mushotzky, R.F., Arnaud, K.A. 1992, ApJ, 395, 275

Edelson, R., Nandra, K. 1999, ApJ, 514, 682

Esin, A.A., Narayan, R., Cui, W., Grove, E.C., Zhang, S.-N. 1998, ApJ, 505, 854

Ford, E.C. et al. 1999, ApJ, 512, L31

Ford, E.C. 2000, ApJ, submitted astro-ph/0002052

Galeev, A.A., Rosner, R., Vaiana, G.S. 1979, ApJ, 229, 318

George, I.M., Fabian, A.C. 1991, MNRAS, 249, 352

Gierliński, M. et al. 1997, MNRAS, 288, 958

Gilfanov, M. et al. 1995, in The Lives of the Neutron Stars, NATO C 450. Kluwer Academic Publishers, Dordrecht, p.331

Gilfanov, M., Churazov, E., Revnivtsev, M. 2000, A\&A, in press astro-ph/9910084)

Grebenev, S.A. et al. 1993, ApJS, 97, 281

Grebenev, S.A., Sunyaev, R.A., Pavlinsky, M.N. 1997, Adv. Space Res., 19, (1)15

Grove, J.E. et al. 1997, in Proceedings of 4th Compton Symposium, AIP Conf. Proc. Vol. 410, AIP, New York, p.122

Grove, J.E. et al. 1998, ApJ, 502, L45

Haardt, F., Maraschi, L. 1993, ApJ, 413, 507

Haardt, F., Maraschi, L., Ghisellini, G. 1994, ApJ, 432, L95

Hasinger, G. 1987, in The Origin and Evolution of Neutron Stars, IAU Symp. 125, D. Reidel Publ. Co., Dordrecht, 333

Hua, X.-M., Kazanas, D., Cui, W. 1999, ApJ, 512, 793

Hynes R.I., O’Brien, K., Horne, K., Chen, W., Haswell, C.A. 1998, MNRAS, 299, L37 
Ichimaru, S. 1977, ApJ, 214, 840

Johnson, W.N. et al. 1997, in Proceedings of 4th Compton Symposium, AIP Conf.Proc. Vol. 410, AIP, New York, p.283

Kaaret, P., Piraino, S., Ford, E.C., Santangelo, A. 1999, ApJ, 514, L31

Kazanas, D., Hua, X.-M., Titarchuk, L. 1997, ApJ, 480, 735

Lamb, F.K. 1989, in Proc. 23rd ESLAB Symp. on Two-Topics in X-ray Astronomy, ESA SP-296, p. 215

Lee, J.C. et al. 1999, MNRAS, submitted astro-ph/9909239

Lewin, W.H.G., van Paradijs, J., van der Klis, M. 1988, Space Sci. Rev., 46, 273

Lochner, J.C., Swank, J.H., Szymkowiak, A.E. 1991, ApJ, 376, 295

Maccarone, T. et al. 2000, in preparation

Magdziarz, P., Zdziarski, A.A. 1995, MNRAS, 273, 837

Malzac, J., Jourdain, E. 2000, A\&A, submitted

Miller, G.S., Lamb, F.K. 1992, ApJ, 388, 541

Miller, K.A., Stone, J.M. 2000, ApJ, in press astro-ph/9912135)

Mitsuda, K., Dotani, T. 1989, PASJ, 41, 557

Miyamoto, S., Kitamoto, S. 1989, Nature, 342, 773

Miyamoto, S., Iga, S., Kitamoto, S., Kamado, Y. 1993, ApJ, 403, L39

Miyamoto, S., Kitamoto, S., Mitsuda, K., Dotani, T. 1988, Nature, 336, 450

Miyamoto, S. et al. 1991, ApJ, 383, 784

Miyamoto, S. et al. 1992, ApJ, 391, L21

Morgan, E.H., Remillard, R.A., Greiner, J. 1997, ApJ, 482, 993

Mushotzky, R.F., Done, C., Pounds, K.A. 1993, Ann. Rev. Astron. Astrophys., 31, 717

Nandra, K., Pounds, K.A. 1994, MNRAS, 268, 405

Narayan, R., Mahadevan, R., Quataert, E. 1998, in Theory of Black Hole Accretion Discs, Cambridge Univ. Press, Cambridge, p.148

Nolan, P.L. et al. 1981, ApJ, 246, 494

Nowak, M.A. et al. 1999a, ApJ, 510, 874

Nowak, M.A., Wilms, J., Dove, J.B. 1999b, ApJ, 517, 355

Nowak, M.A. et al. 1999c, ApJ, 515, 726

Olivie, J.-F., Barret, D. 2000, these proceedings

Page, C.G. 1985, Space Sci. Rev., 40, 387

Papadakis, I.E., Lawrence, A. 1995, MNRAS, 272, 161

Payne, D.G. 1980, ApJ, 237, 951

Pounds, K.A. et al. 1990, Nature, 344, 132

Poutanen, J. 1998, in Theory of Black Hole Accretion Discs, Cambridge Univ. Press, Cambridge, p. 100

Poutanen, J., Fabian, A.C. 1999a, MNRAS, 306, L31

Poutanen, J., Fabian, A.C. 1999b, in High Energy Processes in Accreting Black Holes, ASP Conf. Series Vol. 161, ASP, San Francisco, p.135

Poutanen, J., Svensson, R. 1996, ApJ, 470, 249

Poutanen, J., Krolik, J.H., Ryde, F. 1997, MNRAS, 292, L21

Poutanen, J., Nagendra, K.N., Svensson, R. 1996, MNRAS, 283, 892

Priedhorsky, W. et al. 1979, ApJ, 233, 350 
Psaltis, D., Belloni, T., van der Klis, M. 1999, ApJ, 520, 262

Reig, P. et al. 2000, ApJ, submitted astro-ph/0001134

Revnivtsev, M. 1999, PhD thesis, Space Research Institute, Moscow astro-ph/9912556

Revnivtsev, M., Borozdin, K., Priedhorsky, W.C., Vilhlinin, A. 2000, ApJ, 530, in press (astro$\mathrm{ph} / 9905380$

Romanova, M. M. et al. 1998, ApJ, 500, 703

Shapiro, S.L., Lightman, A.P., Eardley, D.N. 1976, ApJ, 204, 187

Shibazaki, N. et al. 1988, ApJ, 331, 247

Smith, D.M. et al. 1997, ApJ, 489, L51

Smith, I.A., Liang, E.P. 1999, ApJ, 519, 771

Stern, B.E., Poutanen, J., Svensson, R., Sikora, M., Begelman, M.C. 1995, ApJ, 449, L13

Stern, B.E., Svensson, R. 1996, ApJ, 469, L109

Sunyaev, R.A., Titarchuk, L.G. 1980, A\&A, 86, 121

Sunyaev, R.A., Trümper, J. 1979, Nature, 279, 506

Svensson, R. 1996, A\&AS, 120C, 475

Takizawa, M. et al. 1997, ApJ, 489, 272

Tanaka, Y., Lewin, W.H.G. 1995, in X-ray binaries, Cambridge Astrophysics Series, vol. 26, Cambridge University Press, Cambridge, 126

Terrell, N. J. Jr. 1972, ApJ, 174, L35

van der Hooft, F. et al. 1999a, ApJ, 513, 477

van der Hooft, F. et al. 1999b, ApJ, 519, 332

van der Klis, M. et al. 1987, ApJ, 319, L13

van der Klis, M. 1989, in Timing Neutron Stars, NATO ASI C 262. Kluwer Academic Publishers, 27

van der Klis, M. 1995a, in The Lives of the Neutron Stars, NATO ASI C 450. Kluwer Academic Publishers, 301

van der Klis, M. 1995b, in X-ray binaries, Cambridge Astrophysics Series, vol. 26, Cambridge University Press, Cambridge, p.252

Vaughan, B.A., Nowak, M.A. 1997, ApJ, 474, L43

Vaughan, B. et al. 1994, ApJ, 421, 738

Vaughan, B. et al. 1997, ApJ, 483, L115 (erratum 1998, ApJ, 509, L145)

Vaughan, B.A. et al. 1999, A\&A, 343, 197

Velikhov, E.P. 1959, Sov. Phys. JETP, 36, 995

Vilhu, O., Nevalainen, J. 1998, ApJ, 508, L85

Wardzinski, G., Zdziarski, A.A. 2000, MNRAS, in press astro-ph/9911126

Wijnands, R., van der Klis, M. 1999, ApJ, 514, 939

Wijnands, R., Homan, E., van der Klis, M. 1999, ApJ, 526, L33

Yoshida, K. et al. 1993, PASJ, 45, 605

Zdziarski, A.A. 1998, MNRAS, 296, L51

Zdziarski, A.A., Johnson, W.N., Poutanen, J., Magdziarz, P., Gierliński, M. 1997, in The Transparent Universe, ESA SP-382, p.373

Zdziarski, A.A. 1999, in High Energy Processes in Accreting Black Holes, ASP Conf. Series Vol. 161, p.16

Zdziarski, A.A., Lubiński, P., Smith, D.A. 1999, MNRAS, 303, L11 